\title{
Hosting the plant cells in vitro: recent trends in bioreactors
}

\author{
Milen I. Georgiev • Regine Eibl • Jian-Jiang Zhong
}

Received: 3 February 2013 /Revised: 24 February 2013 / Accepted: 26 February 2013 /Published online: 16 March 2013

(C) Springer-Verlag Berlin Heidelberg 2013

\begin{abstract}
Biotechnological production of high-value metabolites and therapeutic proteins by plant in vitro systems has been considered as an attractive alternative of classical technologies. Numerous proof-of-concept studies have illustrated the feasibility of scaling up plant in vitro system-based processes while keeping their biosynthetic potential. Moreover, several commercial processes have been established so far. Though the progress on the field is still limited, in the recent years several bioreactor configurations has been developed (e.g., socalled single-use bioreactors) and successfully adapted for growing plant cells in vitro. This review highlights recent progress and limitations in the bioreactors for plant cells and outlines future perspectives for wider industrialization of plant in vitro systems as "green cell factories" for sustainable production of value-added molecules.
\end{abstract}

All authors equally contributed to this review.

M. I. Georgiev $(\bowtie)$

Department of Applied Biotechnologies, Institute of Microbiology,

Bulgarian Academy of Sciences, 139 Ruski Blvd,

4000 Plovdiv, Bulgaria

e-mail: milengeorgiev@gbg.bg

R. Eibl

School of Life Sciences and Facility Management, Institute of Biotechnology, Zurich University of

Applied Sciences and Arts, Grüental,

8820 Wädenswil, Switzerland

J.-J. Zhong

State Key Laboratory of Microbial Metabolism, and Laboratory of Molecular Biochemical Engineering, School of Life Sciences \&

Biotechnology, Shanghai Jiao Tong University,

800 Dong-chuan Road,

Shanghai 200240, China
Keywords Bioreactor design - Differentiated plant in vitro systems $\cdot$ Culture mode $\cdot$ Plant cell culture $\cdot$ Signal transduction engineering $\cdot$ Single-use bioreactors

\section{Introduction and historical background: from simple jars to single-use bioreactors}

On May 29, 1956, Routien and Nickell (in association with Pfizer \& Co Inc, NY, USA) were granted the first patent for the cultivation of plant cells in vitro (Routien and Nickell 1956). In the patent, entitled "Cultivation of plant tissue", they described the submerged cultivation of tissues of several plant species (e.g., sorrel, sweet clover, and Agave as well as crown galls - plant culture system, obtained via genetic transformation with Ti-plasmid fragment of Agrobacterium tumefaciens -of several plants) in simple 20-L carboy systems (Routien and Nickell 1956). In the early 1960s, the National Aeronautics and Space Administration initiated a research program on plant cells culture for regenerative life support systems. Plants and the relevant cell cultures were grown under various conditions of microgravity (space shuttles, parabolic flights, biosatellites, the orbital stations Salyut and Mir) along with ground studies using rotating clinostat vessels (Sajc et al. 2000). Later on, a conical glass-made V-shape reactor (specially designed for plant cell culture) was introduced (Veliky and Martin 1970) along with the successful adaptation of the stirred-jar reactors for plant cell suspensions by several research groups (Misawa 1994). At the end of the 1970s, the concept of high shear sensitivity of plant cells was developed; and for a decade, only air-lift bioreactors were considered suitable for their cultivation (Verpoorte et al. 2002; Georgiev et al. 2009b). However, several industrialscale processes developed (see below) with stirred-tank bioreactors (STR), subsequently challenged such perceptions (Zhong 2002; Georgiev et al. 2009b). Nowadays, plenty 
designs and configurations of bioreactors for plant in vitro systems are widely available (Eibl and Eibl 2008; Eibl et al. 2009a, b; Georgiev et al. 2009a, b; Georgiev et al. 2011; Zhong 2011). Therefore, it would be difficult, if not impossible, to select the "best" bioreactor design for cultivating different plant in vitro systems (Georgiev et al. 2009a).

In general, the basic function of a bioreactor is to provide optimum conditions for cell physiology and metabolism by regulating various environmental (chemical and physical) factors. The design and selection of each bioreactor are unique but there are some basic principles. Generally, the main criteria for designing plant cell/organ culture bioreactor should consider adequate oxygen transfer, low shear stress, and good mixing (Zhou et al. 2010; Zhong 2011).

In considering the reactor design and selection, nutrients must be effectively provided to the cells. Cell growth and product formation kinetics should be assessed so that an optimal environmental condition can be defined and an operational mode can be determined. Transport phenomena, including mixing, shear force, and oxygen transfer, should be studied so as to define the criteria for bioreactor design and scale-up. Operating parameters, such as temperature, $\mathrm{pH}$, dissolved oxygen concentration, and substrate concentrations, should be easy to control and monitor. In addition, the bioreactor should be as simple and inexpensive as possible and it should easily operate while guaranteeing longterm sterility. Most often it is impossible to meet all the requirements, and thus, some compromise must be made. It is very important to give a balanced consideration between mixing and mass transfer requirements, and the shear sensitivity of cells in the design of large-scale bioreactor systems for hosting plant cells in vitro.

This review summarizes the bioreactor-related aspects of plant cell, tissue/organ culture with particular emphasis on: hardware configuration set-up for plant in vitro systembased bioprocesses, operational mode optimization, and the development of new generation of bioreactors for their cultivation. We also attempted to outline future perspectives for wider industrialization of plant in vitro systems as green cell factories for value-added products.

\section{Recent advances in bioreactors for suspended plant cells: hardware configuration and operational mode}

\section{Bioreactor hardware}

Stirred-tank bioreactor is most widely used bioreactor, and it has advantages such as easy scale-up, good fluid mixing and oxygen transfer ability, alternative impellers, and easy compliance with current Good Manufacture Practices (cGMP) requirements. However, this type of bioreactors also has limitations, such as high power consumption, high shear, and the concerns about sealing and stability of shafts in tall bioreactors. Compared to microbes, plant cells are shear sensitive so considerable efforts are required to modify and optimize the impeller system to balance mixing and mass transfer requirements, and potential damage by hydrodynamic force. Numerous modifications of conventional STRs have been made by designing new impellers (e.g., Fig. 1; Zhong 2011).

Pneumatically agitated bioreactor (air-lift and bubblecolumn bioreactor) is a type of gas-liquid dispersion reactor consisting of a cylindrical vessel, where compressed air or gas mixture is usually introduced at the bottom of the vessel through nozzles, perforated plates or a ring sparger, for aeration, mixing and fluid circulation, without moving mechanical parts. Such type of bioreactor has some advantages over STR: characterized by a more homogeneous energy dissipation (Merchuk and Gluz 1999), and easy to construct and scale-up with low cost. However, the lack of an impeller also brings disadvantages such as poor fluid mixing for viscous culture and serious foaming under high aeration. Air-lift bioreactors with various configurations have been constructed for use in some plant cell cultures, e.g., 200-L air-lift reactor with modified internal configuration was used for saikosaponin production (Kusakari et al. 2012).

\section{Bioprocess parameters}

As described above, a bioreactor should provide optimum conditions (e.g., temperature, $\mathrm{pH}$, oxygen transfer, mixing, and substrate concentration), in addition to its basic function of containment. Cellular metabolism depends on local concentrations in the reactor, as well as on the physiological status of the cell. For bioreactor operation, cellular metabolism must be considered together with the flow profile and the mass transfer characteristics of the reactor because they interact with each other.

Mixing Adequate mixing is essential to ensure the sufficient supply of nutrients and to prevent toxic metabolites accumulation. Mixing time is a critical parameter to be investigated and evaluated. In cell suspension culture processes, mixing is often evaluated in terms of biological performance such as growth rate and productivity. The control of temperature, $\mathrm{pH}$, and substrate concentration are all dependent on good mixing in the bioreactor. Although it is easy to maintain a homogeneous condition in a small-scale reactor, mixing often becomes one of the constraints during scaleup. In the large-scale bioreactors, poor mixing often leads to undesirable concentration gradients and a decrease in mass transfer efficiency. In shear-sensitive plant cell cultures, mixing cannot be enhanced simply by increasing agitation intensity because excessive agitation would cause 
Fig. 1 Stirred-tank bioreactors (STRs) with modified impellers. a Gate paddle reactor with a spiral sparger. b Large flat-bladed impeller reactor. c Helical ribbon impeller reactor. d Centrifugal impeller bioreactor. 1 stirrer, 2 gas in, 3 head plate, 4 shaft, 5 measuring points for liquid velocity, 6 sparger, 7 blade, 8 draft tube, 9 dissolved oxygen (DO) probe, 10 rotating pan (Adapted from Wang and Zhong 1996a; This material is reproduced with permission of John Wiley \& Sons, Inc.) (a)
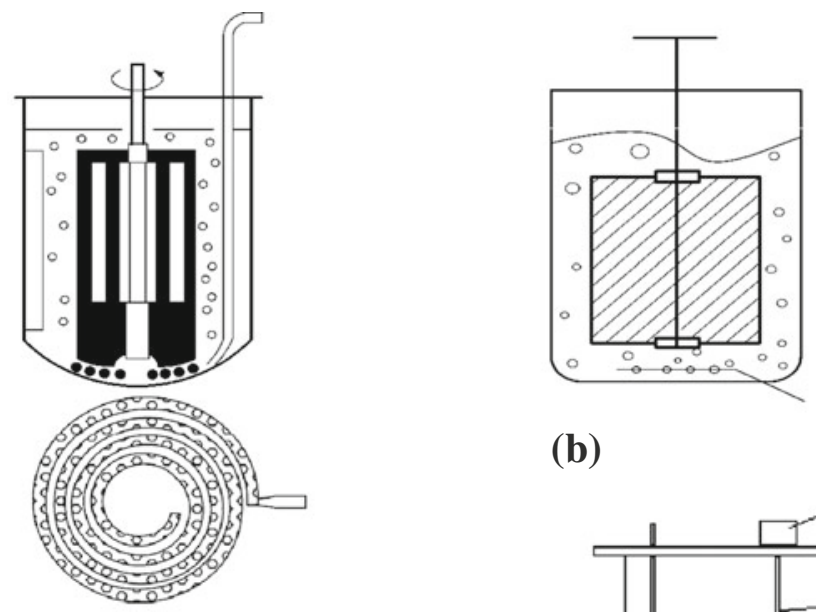

(b)

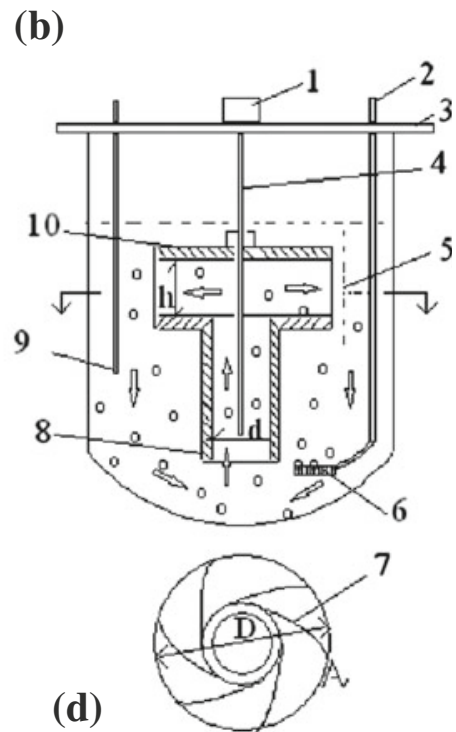

(c)

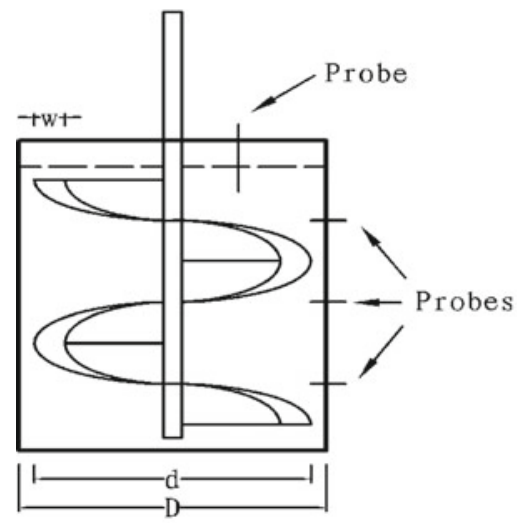

(d) mechanical damage to living cells. A quantitative study on the effect of mixing time on taxoid production in suspension cultures of Taxus chinensis using a centrifugal impeller bioreactor demonstrated the significance of mixing on secondary metabolite production even in a small-scale bioreactor (Zhong et al. 2002).

Oxygen transfer Oxygen transfer is always a concern in aerobic biological systems. Most nutrients required for cellular growth and metabolism are highly soluble in water; sufficient and timely supply of these nutrients can be achieved in a well-mixed reactor. However, oxygen transfer often becomes a limiting step to the optimal performance of biological systems and also for scale-up because oxygen is only sparingly soluble in aqueous solutions. When oxygen supply is limited, both cell growth and product formation would be severely affected. A serious shortage of oxygen can be expected at a high cell density. Aggravating this problem, high cell density often causes the oxygen transfer coefficient to deteriorate. Since volumetric oxygen transfer coefficient $\left(\mathrm{k}_{\mathrm{L}} \mathrm{a}\right)$ is so important in supplying oxygen to the medium, a very critical aspect of reactor design is to achieve a sufficiently high $\mathrm{k}_{\mathrm{L}} \mathrm{a}$, which is affected by many factors (including the geometrical and operational characteristics of the reactor vessel, agitation speed, aeration rate, fluid hydrodynamics, media composition, cell type, morphology, and concentration etc.).

Although the oxygen consumption of plant cells is lower than that of microbes, limitation in oxygen transfer is also a constraining factor for cell cultures at high cell density. Maintaining a suitable oxygen concentration in the culture broth is equally important. The optimal dissolved oxygen concentration may be different for cell growth and product formation (Zhong 2001; Pavlov et al. 2005).

Shear force Compared to microbes, plant cells are much bigger and more sensitive to shear force. Shear has been mainly considered in the literature as a destructive element. A couple of parameters are related to the shear-induced cell death, such as shear stress, shear time, power dissipation, and the growth phase of the cells. Sparging can also cause the shear damage, which can happen at different locations in bioreactor: bubble generation zone at the sparger, the rising zone through the bulk liquid, and the surface of the suspension (either be covered with foam layer or free of foam). To analyze the effects of shear stress on plant cell cultures, a quantitative investigation of the influence of hydrodynamic shear on the cell growth and anthocyanin pigment 
production by Perilla frutescens was demonstrated by using a plant cell bioreactor with marine impeller of larger or smaller diameter (Zhong et al. 1994).

For shear-sensitive cell cultures, therefore, reducing the shear stress intensity by decreasing the agitation speed of the impeller is a general solution. However, this can also bring inadequate mixing and may conflict with enhancing oxygen and heat transfer rates in a high-viscosity cell culture broth. Furthermore, at high biomass concentrations, low agitation rates can also enhance the clumping of cells into cell aggregates of various sizes. However, appropriate bioreactor design and control can minimize shear damage from agitation and aeration. Wang and Zhong successfully designed a novel centrifugal impeller bioreactor (CIB; Fig. 1d) for shear-sensitive biological systems including plant cell cultures (Wang and Zhong 1996a, b). This CIB has been demonstrated to be very successful in high cell density plant cell cultures (Zhang and Zhong 2004; Prakash and Srivastava 2007). In the cultures of Centaurea calcitrapa cells, the key factors improving milk-clotting protease production in a bioreactor were efficient mass transfer and good bulk mixing (without formation of stagnant zones, while a compromise had to be established in relation to hydrodynamic shear conditions; Raposo and Lima-Costa 2012).

Another possibility to overcome the problem of the rising air bubbles is bubble-free aeration using membranes for indirect aeration in which the supply of oxygen is diffusion controlled and no bubbles arise. As the length of the membrane is limited, the average oxygen transfer rate is low. Also, the pressure inside the tubing has limitation. Therefore, this type of bioreactor is quite suitable for small scale cell cultures but limited for large scale (Kretzmer 2002).

\section{Bioreactor operation}

An effective bioreactor operating strategy should provide a high volumetric productivity, which means more products can be formed per unit time per unit bioreactor volume. A major disadvantage of standard batch processes is that significant amount of time is taken by sterilization, filling, emptying, and cleaning of the system. Thus, to enhance the costeffectiveness of plant cell culture processes, various operational modes such as multi-stage batch, fed-batch, single- or multi-stage continuous (chemostat), semicontinuous (drawand-fill or repeated batch), and perfusion (chemostat with cell retention) cultivations have been developed so far. For example, bioreactor operation strategies, such as fed-batch, twostage, etc., were demonstrated to affect the biomass production and secondary metabolite accumulation in bioreactors at commercial scale (Baque et al. 2012). In a pilot scale bioreactor with $10 \mathrm{~m}^{3}$ capacity, a two-stage culture system was applied, aiming to maximize both root biomass and secondary metabolite contents. In the first stage, the adventitious roots are cultured under optimal culture conditions for biomass accumulation followed by physical and chemical elicitations for secondary metabolite accumulation (Paek et al. 2009).

Fed-batch culture Generally, the final product concentration is primarily affected by the specific productivity of cells and integrated cell growth. To overcome nutrient limitation, fedbatch processes have been widely practiced which involves the addition of one or more nutrients continuously or intermittently to the initial medium after the start of cultivation, or from certain point during the batch process. A comparison of different feeding modes is presented in Table 1. Among these, the most popular and successfully used ones are fed-batch and perfusion.

Fed-batch cultures are currently used for most cell culture processes, especially for intracellular products of cell cultures which are stored within the cells. For example, Hu and Zhong (2001) performed fed-batch cultivation of Panax notoginseng cells in an air-lift reactor to study the effects of bottom clearance on cell growth and the production of ginseng saponin and polysaccharide. A fed-batch mode was also developed, in which sucrose was fed just prior to a sharp decrease in the specific oxygen uptake rate (Hu et al. 2001). By applying this feeding strategy to P. notoginseng cell suspensions in a 10-L air-lift bioreactor (Hu et al. 2001) and a 30-L CIB (Zhang and Zhong 2004), a very high biomass productivity of about $1.5 \mathrm{~g} /(\mathrm{L} . \mathrm{d})$ was achieved, and both saponin and polysaccharide productivities were also higher than those in a comparative batch process. Fed-batch culture mode was also demonstrated to be effective for the production of human cytotoxic T-lymphocyte antigen 4-immunoglobulin (hCTLA4Ig) in transgenic rice cell suspension cultures. By performing fed-batch cultivation in a 15-L stirred-tank bioreactor, maximum hCTLA4Ig level was $76.5 \mathrm{mg} / \mathrm{L}$ at day 10 or 1.2 -fold higher compared to that of two-stage culture with medium exchange (Park et al. 2010). In addition, fed-batch operation combined with elicitation was useful in enhancing the production of stilbenes by grape cell culture (Vitis vinifera $\mathrm{cv}$. Barbera) in bioreactors (Ferri et al. 2011).

Continuous and semicontinuous culture Theoretically continuous culture is the most promising mode for obtaining high productivity. In a continuous culture, the nutrients consumed by the cells are continuously replenished by an inflow of fresh medium. A constant inflow of fresh medium is balanced by a constant efflux of spent medium plus cells. Consequently, a steady state will be developed at a dilution rate (equal to outflow rate/volume) less than the maximum specific growth rate of the culture. Under steady state conditions, the average specific growth rate in the culture is identical to the dilution rate. The biomass 
Table 1 Comparison of commonly used modes of feeding based on product yield and process economics

\begin{tabular}{lllll}
\hline Culture mode & Product yield & $\begin{array}{l}\text { Process } \\
\text { manipulation }\end{array}$ & $\begin{array}{l}\text { Cost (capital investment } \\
\text { and labor) }\end{array}$ & Throughput \\
\hline Batch & Low & Low & Low & Low \\
Repeated batch & Low & Medium & Medium & Medium \\
Fed-batch & High & Medium & Low & Low \\
Perfusion & Medium & High & Medium & High \\
Continuous & Low & Medium & Low & Low \\
\hline
\end{tabular}

concentration of the culture is then determined by the concentration of the growth-limiting nutrient in the influent. All culture parameters (biomass, biomass composition, and nutrients) remain constant for prolonged periods of time in a steady state.

An integrated approach was adopted by combining various yield and productivity enhancement strategies, i.e., coupling the mathematical model-derived nutrientfeeding strategy for continuous cultivation with elicitation, precursor addition, and permeabilization, etc. With such an integrated approach, a high yield and high productivity of azadirachtin (a biopesticide) were obtained in suspension cultures of Azadirachta indica (Prakash and Srivastava 2011).

Plant cell culture is an alternative for the production of recombinant human therapeutic proteins because of improved product safety, lower production cost, and capability for eukaryotic post-translational modification. An optimized semicontinuous bioreactor operation was proposed to maximize the production of recombinant human alpha-1antitrypsin (rAAT) glycoprotein by transgenic Nicotiana benthamiana cell culture. Compared to a traditional batch bioreactor operation, a 25-fold increase in extracellular functional rAAT was achieved (Huang et al. 2010).

Perfusion culture Perfusion cultivation is carried out by continuously feeding fresh medium to the reactor and constantly removing the cell-free spent medium while retaining the cells, thus a much higher cell density can be obtained in perfusion cultures compared to continuous ones, as cells are retained within the reactor via a cell retention device. The perfusion rate depends on the cell line demand, the concentration of nutrients in the feed, and the level of toxification. The overall advantage of perfusion cultures is that it requires a smaller scale compared to batch cultures in order to obtain the desired amount of product such as plant secondary metabolite.

In a perfusion bioreactor, Cloutier et al. (2009) performed adaptive medium feeding using model mass balances and estimations. The proposed culture strategy led to a significant increase in both specific production and total production, as compared with a standard batch culture protocol.
Operation combined with signal transduction engineering Signal transduction engineering was proposed and applied to taxuyunnanine $\mathrm{C}$ biosynthesis in suspension cultures of $T$. chinensis (Qian et al. 2004, 2005; Zhou and Zhong 2011a, 2011b). Novel chemically synthesized jasmonates, such as 2-hydroxyethyljasmonate, 2,3-dihydroxypropyljasmonate, pentafluoropropyl jasmonate, 2-(2,6-dichloro-pyridine-4carbonyloxy)-ethyl jasmonate, and trifluoroethyl salicylate, were proved to be powerful inducing signals for plant secondary metabolism in both shake flasks and bioreactors (Qian et al. 2004, 2005), and the signal transduction mechanism and induced biosynthetic genes transcription were elucidated (Hu et al. 2006). Application of inducers or elicitors, such as sugar depletion for recombinant proteins production by rice suspension cells (Liu et al. 2012), jasmonate treatment for resveratrol production (Donnez et al. 2011), and for C-13-labeled trans-resveratrol production (Yue et al. 2011) in grapevine cell cultures, was also reported in various cases. In the cell cultures of Harpagophytum procumbens for production of antiinflammatory phenylethanoid glycosides, the relationship between metabolite production and stress-related hormones including abscisic, jasmonic, and salicylic acids was discussed with respect to relative stress levels in the different cultivation systems (Georgiev et al. 2011).

Industrial operation using plant cells Include those of paclitaxel, shikonin, berberine, ginseng, Echinacea polysaccharides, and several therapeutic proteins production, among others (additional examples can be found in recent review by Wilson and Roberts 2012). With acquisition of highly productive cell lines and establishment of culture conditions and procedures that ensure maximum productivity of cells, in 1983 the Mitsui Petrochemical Industries (now Mitsui Chemicals) succeeded in two-stage suspension cultures of Lithospermum erythrorhizon in a 750-L air-lift bioreactor yielding 1.4-2.3 g/L shikonin within 23 days. Later, in a similar approach by lowering the production cost, they also established an industrial production process of berberine production from Coptis japonica cell cultures at a scale up to $4 \mathrm{~m}^{3}$ with its highest berberine yield of $3.5 \mathrm{~g} / \mathrm{L}$. In 1989, Nitto Denko Corporation developed cell suspension culture of Panax ginseng at a scale of $25 \mathrm{~m}^{3}$. Cell 
suspension cultures of Taxus species were commercialized by Phyton GmbH (Ahrensburg, Germany), the largest cGMP plant cell culture facility in the world with bioreactors up to $75 \mathrm{~m}^{3}$ in size (Zhong 2002; Georgiev et al. 2009b; Onrubia et al. 2013). The Phyton Biotech (Ithaca, NY) is a global provider of chemotherapeutic agents including paclitaxel, docetaxel APIs (active pharmaceutical ingredients) and taxane intermediates. The cell culture-based paclitaxel production was also succeeded by Samyang Genex Corporation (Taejon, South Korea) at a ton scale (Zhong 2002). All the above successful industrialization cases illustrate the significance of suitable bioreactors hardware configuration and optimized operational modes for suspended plant cells to achieve maximal production of target secondary metabolites.

\section{Culturing uncultivable: bioreactors for differentiated plant in vitro systems}

Differentiated plant in vitro systems include adventitious and hairy root culture (obtained via genetic transformation with T-DNA of Agrobacterium rhizogenes), shoot culture, and plantlets. Micropropagation of plantlets and respective bioreactor configurations are beyond the scope of this review and, thus will not be further considered here. Although, the large scale production of high-value molecules and their commercialization by plant cell cultures has been established, there are several shortcomings such as the poor productivity, instability of dedifferentiated cultures, as well as some valuable compounds are not synthesized in the dedifferentiated cells (Verpoorte et al. 2002; Baque et al., 2012). Differentiated plant in vitro systems (= plant tissue and organ culture) became an attractive platform for bioproduction of plant-derived metabolites and therapeutic proteins (mainly hairy root culture systems; Georgiev et al. 2012a). Their several advantages, such as genetic and biochemical stability and capacity for organogenesis-associated synthesis of metabolites brought plant tissue and organ culture to the foreground.

The physiology and morphology of differentiated plant in vitro systems demand special consideration with regard to effective bioreactor configuration providing a low-shear environment for tissue growth and reducing mass transfer limitations (formation of strong nutrient and oxygen gradients in the tissue present major a challenge to scale-up in bioreactors) in densely packed plant tissue (non-homogeneous growth) beds (Eibl and Eibl 2008; Georgiev et al. 2012a). This nonhomogeneous growth of differentiated plant in vitro systems is rather challenging, as the transfer of the plant tissue inocula from small reactors to large-scale reactors cannot be preformed pneumatically (as usually done in microbial and plant cell culture-based processes; Georgiev et al. 2012a). Monitoring of the plant tissue growth in bioreactors is also a challenging issue (Georgiev et al. 2012a). Indirect estimates of the tissue growth (either in off-line mode via sampling or in online mode) can be obtained through measuring conductivity, osmolarity, and redox potential of the culture medium (Georgiev et al. 2007), though more reliable and accurate methods are continuously sought.

Diverse bioreactor configurations have been used for culturing differentiated plant in vitro systems, including mechanically driven systems (e.g., STR, wave-mixed reactors, and rotating drum reactors), pneumatically driven reactors (e.g., air-lift reactors and bubble-column reactors), and bed reactors (e.g., mist reactors and trickle-bed reactors). As mentioned above it is impossible to pick any single ideal type of bioreactor system for culturing plant tissue and organ cultures for all purposes.

Mechanically driven bioreactors The use of ordinary STR systems is, in general, not recommended because of the high stress-sensitivity of these particular in vitro systems (Georgiev et al. 2008, 2010). However slight changes in the STR internal hardware configuration, such as separating the impeller from the plant tissue (using a mesh) or just a simple reduction of the agitation speed has allowed successful cultivation of transformed root cultures of Beta vulgaris (Georgiev et al. 2006) and H. procumbens (Homova et al. 2010) and significant biomass productivity [0.64-0.68 g dry weight/(L.d)].

Pneumatically driven bioreactors Air-lift and bubblecolumn reactors are probably the most frequently used configurations for hosting differentiated plant in vitro systems. In bubble-column cultivation systems, the sole source of agitation is the pneumatic power input provided by isothermal expansion of the sparged gas (Perez et al. 2006). The air bubbles type of aeration significantly reduces the cells' exposure to local zones of high shear stress and therefore ensures both homogenization of the medium and the mass transfer of oxygen into the liquid medium. Hairy root culture-based bioprocesses (e.g., production of bioactive betalain pigments by $B$. vulgaris and anti-inflammatory phenylethanoid glycosides by $H$. procumbens) were successfully developed in 3-L bubble-column bioreactors (Fig. 2a), operated under batch and fed-batch operation mode (Pavlov et al. 2007; Ludwig-Mueller et al. 2008). Moreover, the levels of stress-related hormones (e.g., abscisic acid, salicylic acid, jasmonic acid, and aminocyclopropane carboxylic acid) were significantly lower in bubble-column bioreactor hairy root culture of $H$. procumbens than in the respective shake-flask culture, which presumably indicates 

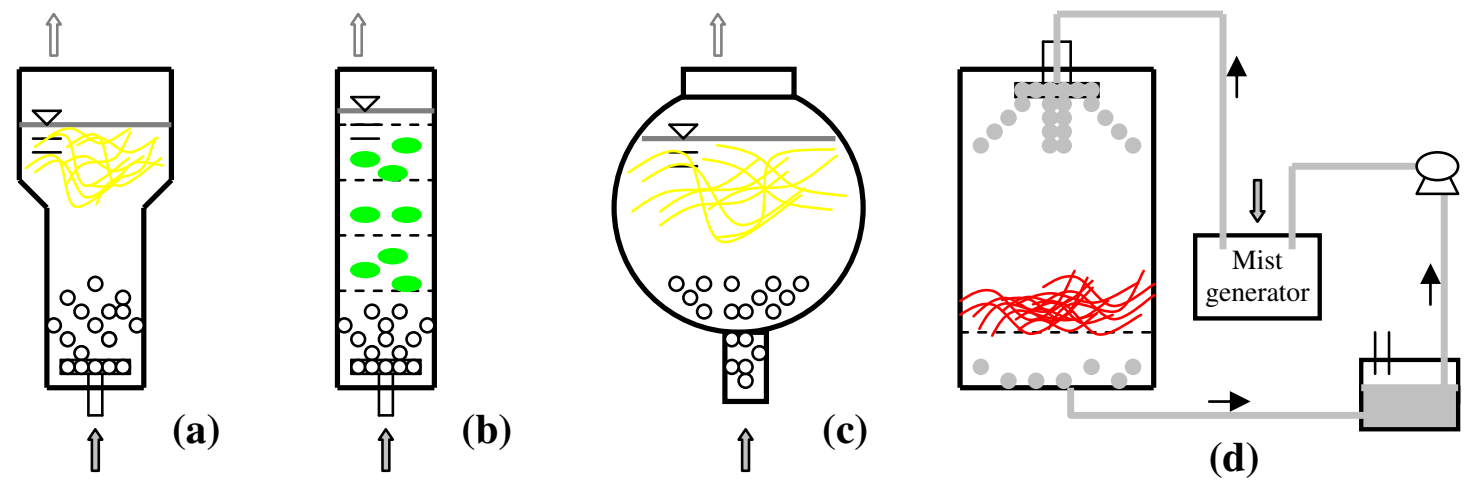

(b)

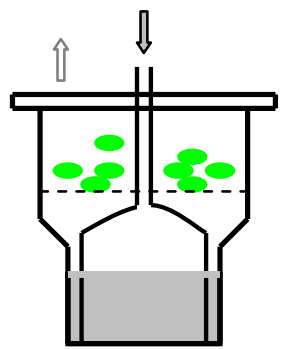

(f)

Inlet air

Gas exhaust (c)

(d)
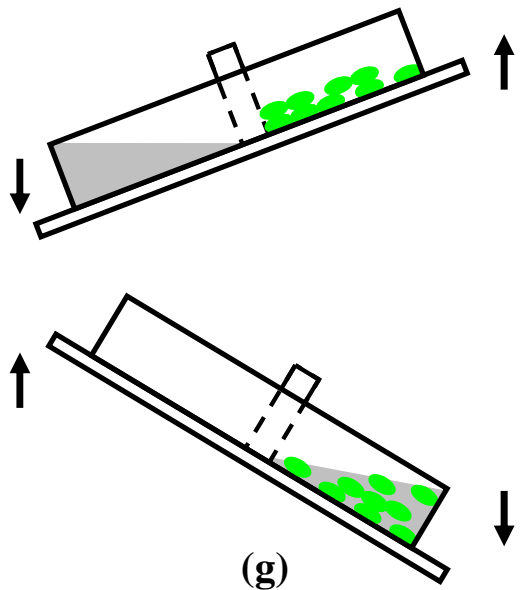

Fig. 2 Schematic diagrams of reactor configuration for differentiated plan in vitro systems. a Bubble-column bioreactor. b Modified column bioreactor with internal sections (Georgiev et al. 2012b). c Balloon type bubble bioreactor (BTBB; Baque et al. 2012). d Mist bioreactor (Kim et al. 2002; Sivakumar et al. 2010). e Trickle-bed bioreactor (Ramakrishnan and Curtis 2004). f RITA system (Georgiev et al. 2008; Ivanov et al. 2011). g BioMINT reactor (Roberts et al. 2006) the low-stress environment of this type of reactors (LudwigMueller et al. 2008). A modification of air-lift reactor, named balloon-type bubble bioreactor (BTBB; Fig. 2c), was found very suitable for large-scale biomass and metabolite production by adventitious root culture. The bioreactor high suitability was proven in several studies, at different scales (from $3 \mathrm{~L}$ to $10 \mathrm{~m}^{3}$ ), with adventitious root cultures of Panax ginseng, Hypericum perforatum, Morinda citrifolia, and Echinacea species (Baque et al. 2012 and the literature cited therein). In a recent study, Georgiev et al. (2012b) reported successful cultivation of Leucojum aestivum shoot culture in an advanced modified glass-column bioreactor with internal sections (Fig. 2b) for production of galanthamine (a reversible inhibitor of acetylcholinesterase, currently used in the clinical practice to treat mild-tomoderate stage of Alzheimer's disease). The introduction of internal sections resulted in reduced problems with flotation of the shoots and allowed efficient stream ways of the fluids. Under optimal culture conditions in the reactor $\left(22^{\circ} \mathrm{C}\right.$ and $18 \mathrm{~L} /(\mathrm{L} . \mathrm{h})$ flow rate of inlet air), highest amounts of shoot biomass [ $>20 \mathrm{~g} / \mathrm{L}$; productivity of $0.57 \mathrm{~g} /(\mathrm{L} . \mathrm{d})]$ and galanthamine $(1.7 \mathrm{mg} / \mathrm{L})$ were observed. The construction of the bioreactor from glass also extends the range of cultivation to photomixotrophic or phototrophic plant tissue and organ culture.

Bed bioreactors Mist and trickle-bed reactors seem to offer optimal environment for culturing differentiated plant in vitro systems. Gas-phase bed reactors can virtually eliminate any oxygen deficiency in high-bed densities, while providing a low shear stress environment (Kim et al. 2002). Mist reactors (Fig. 2d) are a type of gas-phase reactors in which thin films of nutrients solution are sprayed by ultrasonic systems onto the surfaces of plant tissue and organs, collected at the bottom of the reactors, and thus recycled continuously through the system (Kim et al. 2002; Georgiev et al. 2012a). In experiments performed with hairy roots of Artemisia annua and Nicotiana tabacum, at scale of 1.5-4-L mist reactors, biomasses productivity of $0.37-$ $0.38 \mathrm{~g}$ dry weight/(L.d) was achieved (Kim et al. 2002; 
Liu et al. 2009). Similar biomass productivity was reached when the Arachis hypogaea hairy root-based process was further up-scaled to 20-L mist bioreactor (several parameters as misting duty cycle, culture medium flow rate, and timing of when flow rate was increased were optimized; Sivakumar et al. 2010). Moreover, the mist bioreactor design was also found to be advantageous for $A$. апnиа shoot cultures. The accumulated dry weight of $A$. annua shoots and artemisinin (a sesquiterpene lactone with antimalarial properties) production in the mist bioreactor were found significantly higher than those observed in both the modified airlift bioreactor and the multi-plate radius-flow bioreactor (Liu et al. 2003). Another bed reactor system, proven to be very suitable for culturing plant tissue and organ cultures is the trickle-bed reactor design (Fig. 2e). Hairy root cultures of Hyoscyamus muticus grown in 14-L trickle-bed reactor showed very high growth [biomass productivity reached $1.45 \mathrm{~g} /(\mathrm{L} . \mathrm{d})]$ and final mass densities significantly exceeding previously reported values $(>36 \mathrm{~g} / \mathrm{L})$. Moreover, based on the preliminary design calculations, a conclusion that scale-up to at least $10 \mathrm{~m}^{3}$ would be feasible was drawn (Ramakrishnan and Curtis 2004).

Temporary immersion systems These have been originally developed for plant micropropagation purposes. However, their several advantages, such as reduced hyperhydricity and lower consumable costs, made them attractive for differentiated plant in vitro systems cultivation (Georgiev et al. 2007). Several temporary immersion systems were therefore designed and successfully applied, e.g., RITA ${ }^{\circledR}$ systems (Fig. 2f) for H. procumbens (Georgiev et al. 2008) hairy roots and L. aestivum shoot cultures (Ivanov et al. 2011), and BioMINT reactor (Fig. 2g; Roberts et al. 2006). Although, the RITA ${ }^{\circledR}$ systems (about $0.2 \mathrm{~L}$ ) and BioMINT reactor $(1.2 \mathrm{~L})$ are still of laboratory scale, the concept of temporary immersion systems can be further efficiently extended to a large-scale volumes.

Large scale plant tissue and organ culture processes Industrialization of differentiated plant in vitro systembased processes and development of economically viable commercial processes are not yet well developed, mainly due to the morphological features of plant tissue and organ cultures and the resultant challenges. However, the recently developed commercial system (at $10 \mathrm{~m}^{3}$ scale, involving BTBB) for biomass and bioactive ginsenoside production from $P$. ginseng adventitious roots (an average production of ginseng biomass is 45 tons fresh weight per year) by CBN Biotech Company, South Korea (Baque et al. 2012) is a good example for the feasibility of plant tissue and organ cultures for sustainable mass production of high-value compounds.

\section{Single-use (disposable) systems: new generation of bioreactors for plant cells}

Single-use bioreactors (also often referred to as disposable bioreactors) have cultivation containers, consisting of US Food and Drug Administration-approved plastics (Eibl et al. 2011a; Vanhamel and Masy 2011), and are typically used once. They have become increasingly accepted for biotechnological processes at small and medium size scale during the past ten years. This trend can be explained by the advantages they offer: reduced contamination rates, savings in time and costs, and reduced waste and environmental impact. Different studies (Rao et al. 2009; Pietrzykowski et al. 2011; Krishnan and Chen 2012; Rader and Langer 2012) demonstrated that these advantages prevail, although limitations covering undesirable influences on product quality from leachables/extractables and lack of reliable singleuse on-line sensors currently exist. The advantages arise from the cultivation container, which is either a rigid vessel or a flexible multilayer bag fixed, stabilized and shaped by a supporting container. The cultivation container is provided by the vendor in a sterile state and is discarded after completion of the bioprocess, once it has been decontaminated. Cost savings of 20-30\%, which have been reported, can only be achieved when high-value products are being manufactured. The reason for this is that the GMP-compliant cultivation containers are very expensive. As a result, single-use bioreactors are most frequently used for mammalian and insect cellbased antibody and vaccine production, for which the majority of single-use bioreactors were originally developed (Brod et al. 2012).

Although in processes aimed at development and production of plant cell-based high-value products, reusable bioreactors made from glass or stainless steel have to date been predominantly, bioreactors with plastic cultivation containers are not uncommon (Eibl et al. 2009a; Weathers et al. 2010). They are used for cultivation of plant cell suspension and organ cultures, including hairy roots, shoots, and plantlets. Here, it can be differentiated between plastic cultivation containers intended for multiple usage (multi-usable bioreactors) or single usage (single-use bioreactors). Multi-usable bioreactors, such as the previously mentioned temporary immersion bioreactors, the RITA ${ }^{\circledR}$ system (Alvard et al. 1993; Etienne and Berthouly 2002; Georgiev et al. 2008; Ivanov et al. 2011; Watt 2012), and the BioMint (Roberts et al. 2006), are operated using a non-instrumented, low-cost plastic container. In addition to hairy root cultivation, they are commonly used for plant micropropagation and must always be autoclaved (by the user) before use. Further examples of multi-usable bioreactors for hairy root cultures (e.g., from $P$. ginseng and Ocimum basilicum) are the ROOTec Mist bioreactor (Wink et al. 2005; Eibl et al. 2012) and the Weathers' system (Kim et al. 2002; Liu et al. 2009; Sivakumar et al. 2010). 
In general, an exact classification of multi-usable and single-use systems is difficult for plant cell bioreactors which are commercially unavailable. In particular, that is the case for the special designs, such as the Box-in-Bag bioreactor, the Wave-and-Undertow bioreactor, and the Slug Bubble bioreactor (thoroughly discussed in Girard et al. 2006; Ducos et al. 2009; Eibl et al. 2011b), the Plasticlined bioreactor (Hsiao et al. 1999; Curtis 2004), and the Protalix bioreactor (Shaaltiel et al. 2010; Wilson and Roberts 2012). Their specifications are ambiguous and to some extent inconsistent. This is, therefore, the reason why only commercially available single-use bioreactors for plant cells are examined in this review.

For milliliter-scale processes, non-instrumented, singleuse versions of rigid, centrifuge-like tubes, and shake flasks that require a shaking incubator for optimum growth and production have proved themselves in screening studies. For example, Raven et al. (2011) and Brändli et al. (2012) demonstrated the advantages of using the orbitally shaken CultiFlask disposable bioreactor (also known as the TubeSpin) for parameter scouting in M12 antibody production using N. tabacum Bright-Yellow-2 (BY-2) cell suspension culture.

So-called single-use bag bioreactors are used for liter scale processes. Table 2 gives an overview of commercially available types that are suitable for growing plant cells including suspension cells, hairy roots and embryogenic cultures. All the systems are mechanically driven. The single-use cultivation containers are oscillated/wave-mixed, oscillated with vibrating disks, stirred, or more recently, orbitally shaken. Schematic diagrams for these single-use bag bioreactors are shown in Fig. 3. It is worth mentioning that the first benchtop scale, single-use bioreactor for plant cells (e.g., the Life Reactor; Ziv et al. 1998; Ziv 2005), introduced to the market by Osmotek in the early 1990s, is no longer available.

Wave-mixed, single-use bioreactors can be found in the widest range of applications. Mibelle Biochemistry (Switzerland) and Sederma (France) produce substances for cosmetics (e.g., PhytoCellTec Argan, Solar Vitis, Malus domestica, Alp Rose, or RESISTEM), by growing plant cells suspension cultures in the BIOSTAT CultiBag RM reactors. Using the same bioreactor system, Greenovation successfully expressed numerous therapeutic proteins (e.g., recombinant human asialo-erythropoietin) based on Physcomitrella patens suspension cells (www.greenovationcom/bryotechnology.html; accessed on 27 October 2012).

Due to the wave movement, bubble-free oxygen is introduced into the culture broth from the bag's headspace in every wave-mixed bioreactor. Fluid flow, mixing time, oxygen mass transfer rate, and shear stress acting on cells are defined by the propagation of the wave within the bag.
Shear stress patterns are homogeneous, and foaming and flotation are negligible in wave-mixed bioreactors (Eibl et al. 2009b). In processes with $H$. muticus hairy roots, it has been possible to increase product quantity if ebb-and-flowoperation can be guaranteed by running the bag with reduced culture volume. In addition, specially designed bags with a screw cap and integrated mesh have been advantageously used for hairy roots, particularly at total bag volumes exceeding $2 \mathrm{~L}$ (Eibl et al. 2012). There are a few restrictions for cultivating plant cells in wave-mixed bioreactors. Mass and energy transfer in culture broths showing non-Newtonian fluid flow behaviors can be severely limited. Scaling-up is complicated due to the fact that the bags are not geometrically similar, although cultivation bags of different sizes do exist for wave-mixed bioreactors. When cultivating phototropic cultures the installed optical sensors have been identified as an issue (bleaching effect). Furthermore, optical $\mathrm{pH}$ sensors do not provide reliable measurements of $\mathrm{pH}$ values below 5.7, which can appear in plant cell cultivations. The last-mentioned limitation applies to all single-use bioreactors in Table 2 that operate with optical sensors.

In contrast to wave-mixed bag bioreactors where power input and resulting shear stress on cells can be regulated via rocking rate and rocking angle, in the case of the Vibromix reactor (www.liquitec.ch/de/ruehrtechnik/vibromixer/; accessed on 27 October 2012), this is performed via motor amplitude and frequency. In order to cultivate $M$. domestica suspension cells more efficiently, bags were developed which contain a sparger in addition to the oscillating disks mounted on a hollow shaft. Because the Saltus Vibromix bioreactor generates a high local power input, it is not recommended for applications involving shear sensitive plant cells.

Today, stirred single-use bioreactors are not relevant for the growth of plant cells, even though Raven demonstrated the suitability of the 50-L S.U.B. for plantibody production using BY-2 suspension cells (Raven et al. 2011). The microsparger of the tested bag configuration caused significant flotation during cultivation, which resulted in product loss. However, cell growth was only slightly lower than in wave-mixed bag bioreactors.

Interestingly, initial cultivations with BY-2 as well as $V$. vinifera suspension cells and tobacco (Basma xanthi) hairy roots in orbitally shaken bag bioreactors indicated promising results. Cell growth and product formation for similar fluid flow conditions are comparable to the results delivered by wave-mixed systems. Maximum biomass concentrations between 380 and $480 \mathrm{~g}$ fresh weight/L were detected after 7 days (Werner et al. 2013). This comes as no surprise since the advantages of wave-mixed single-use bioreactors (homogeneous energy dissipation, reduced foaming and flotation) were also found for orbitally shaken bag bioreactors 


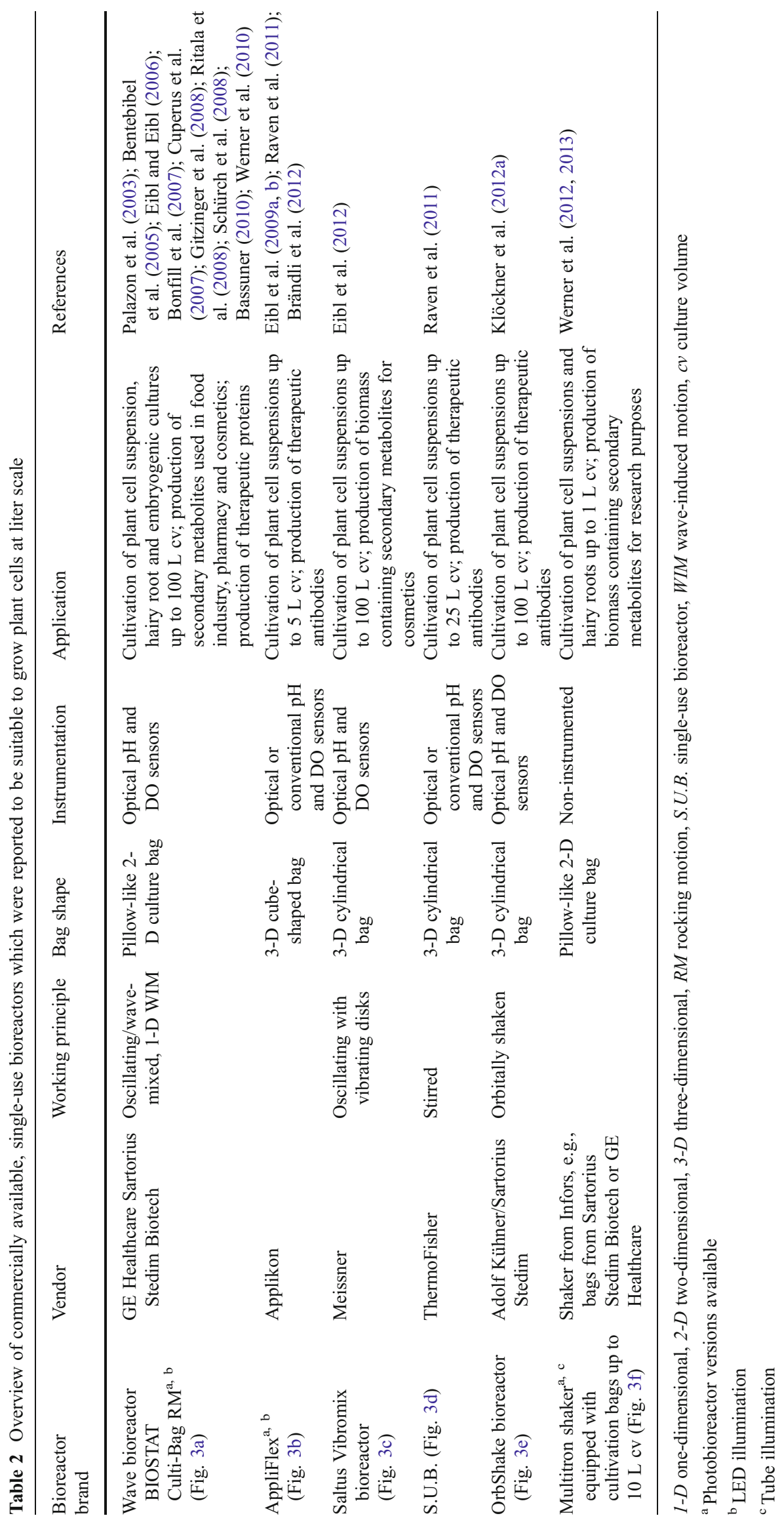




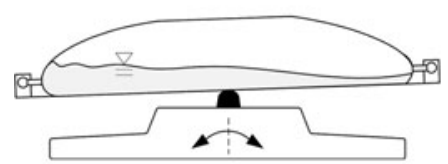

(a)

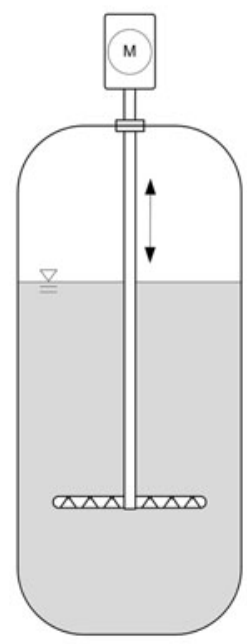

(d)

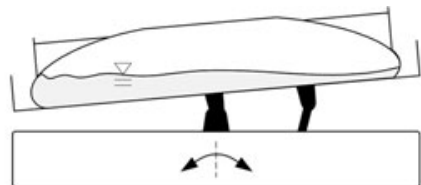

(b)

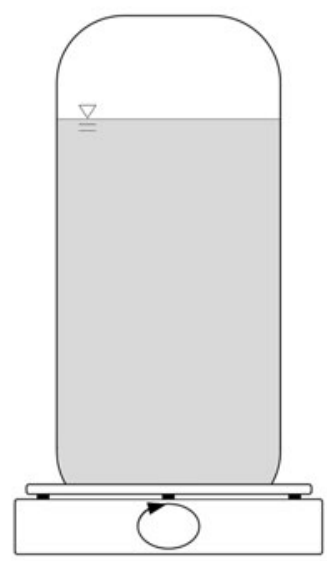

(e)

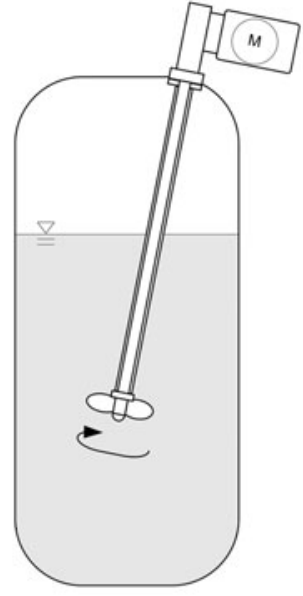

(c)

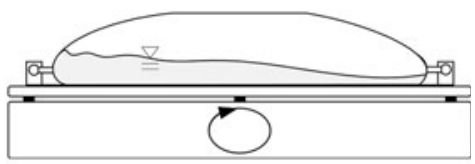

(f)

Fig 3 Schematic diagrams of suitable single-use bioreactors for plant cells. a Wave bioreactor/BIOSTAT CultiBag RM. b AppliFlex. c Saltus Vibromix bioreactor. d S.U.B. e OrbShake bioreactor. f Multitron shaker equipped with cultivation bags

(Klöckner et al. 2012a, b; Werner et al. 2012, 2013). Despite the fact that shaking 2-D cultivation bags in the Multitron Cell is restricted to $10 \mathrm{~L}$ culture volume, scaling-up of 3-D bags (OrbShake bioreactor) to $\mathrm{m}^{3}$ scale is currently under way. Compared to wave-mixed systems, increased power input and easier scaling-up, due to the geometric similarity, are both possible in the OrbShake bioreactor. But it is important to consider the possible occurrence of vortex formation in large scale implementations.

It is obvious that plant cell-based processes have not yet reached the level of significance of mammalian cell-based processes in single-use bioreactors. This is due to the fact that plant cells are less frequently used in high-value product manufacturing. Nevertheless, wave-mixed single-use bioreactors, having a 1-D rocking motion and operating in batch and feeding mode, are already established in the development and production of cosmetic agents and therapeutic proteins.

\section{Conclusions and perspectives}

In the last 15 years, we have witnessed a significant progress in bioreactor configurations (and operational mode) for plant in vitro systems. Numerous proof-of- concept studies have illustrated the feasibility of plant in vitro system-based processes for sustainable mass production of plant derived-molecules and therapeutic proteins, at eco-friendly conditions (green cell factories concept). In the future, further single-use bioreactors such as 2-D (CELL-tainer) and 3-D (XRS bioreactor System) rocking bag bioreactors and orbitally shaken bioreactor systems (OrbShake bioreactor) can be advantageously applied for fast growing plant cells that do not exhibit Newtonian fluid flow behavior. Nevertheless, there are many further challenges that have yet to be investigated (e.g., the secretion of leachables and extractables as cultivation time increases in illuminated phototrophic cultures) which will decide on the success of single-use bioreactors for plant cell cultivations. It is also foreseeable that in the case of increasing demand for single-use bioreactors, plant in vitro system-based processes conceived for multi-usable disposable bioreactors will be redesigned for single-use versions.

Acknowledgments Financial support from the National Science Fund of Bulgaria (grant DO-02-261/2008 to MIG) is greatly appreciated. JJZ appreciates the financial support from the National Natural Science Foundation of China (NSFC) and the SJTU University Distinguished Professorship program as well as excellent collaboration with Prof. Xuhong Qian (ECUST) on novel synthetic elicitors. 


\section{References}

Baque MA, Moh SH, Lee EJ, Zhong JJ, Paek KY (2012) Production of biomass and useful compounds from adventitious roots of highvalue added medicinal plants using bioreactor. Biotechnol Adv 30:1255-1267

Bassuner R (2010) Operating 100 L moss bioreactors secreting mAbs with defined mammalian-like glycosylation. Presentation to EAPB Meeting, 2/24/10, Vienna, Austria

Bentebibel S, Moyano E, Palazon J, Cusido RM, Bonfill M, Pinol MT (2005) Effects of immobilization by entrapment in alginate and scale-up on paclitaxel and baccatin III production in cell suspension cultures of Taxus baccta. Biotechnol Bioeng 89:647-655

Bonfill M, Bentebibel S, Moyano E, Palazon J, Cusido RM, Eibl R, Pinol MT (2007) Paclitaxel and baccatin III production induced by methyljasmonate in free and immobilized cells of Taxus baccata. Biol Plant 51:647-652

Brod H, Vester A, Kauling J (2012) Opportunities and limitations of disposable technologies in biopharmaceutical processes. Chem Ingen Tech 84:633-645

Brändli J, Müller M, Imseng N, Schillberg S, Eibl R (2012) Prozessentwicklung und -übertragung vom $50 \mathrm{~mL}$ auf den $10 \mathrm{~L}$ Massstab. BIOspektrum 2:216

Cloutier M, Chen JK, De Dobbeleer C, Perrier M, Jolicoeur M (2009) A systems approach to plant bioprocess optimization. Plant Biotechnol J 7:939-951

Cuperus S, Eibl R, Hühn T, Amado R (2007) Plant cell culture-based platform: investigating biochemical processes in wine production. BioForum Europe 6:2-4

Curtis WR (2004) Growing cells in a reservoir formed of a flexible sterile plastic liner. United States Patent. 6,709,862 B2

Donnez D, Kim KH, Antoine S, Conreux A, De Luca V, Jeandet P, Clement C, Courot E (2011) Bioproduction of resveratrol and viniferins by an elicited grapevine cell culture in a $2 \mathrm{~L}$ stirred bioreactor. Process Biochem 46:1056-1062

Ducos JP, Terrier B, Courtois D (2009) Disposable bioreactors for plant micropropagation and mass plant cell culture. Adv Biochem Engin/Biotechnol 115:89-115

Eibl R, Eibl D (2006) Design and use of the wave bioreactor for plant cell culture. In: Dutta Gupta S, Baraki Y (eds) Plant tissue culture engineering. Springer, Dordrecht, pp 203-227

Eibl R, Eibl D (2008) Design of bioreactors suitable for plant cell and tissue cultures. Phytochem Rev 7:593-598

Eibl R, Werner S, Eibl D (2009a) Disposable bioreactors for plant liquid cultures at litre-scale: review. Eng Life Sci 9:156-164

Eibl R, Werner S, Eibl D (2009b) Bag bioreactor based on waveinduced motion: characteristics and applications. Adv Biochem Engin/Biotechnol 115:55-87

Eibl D, Peuker T, Eibl R (2011a) Single-use equipment in biopharmaceutical manufacture: a brief introduction. In: Eibl R, Eibl D (eds) Single-use technology in biopharmaceutical manufacture. John Wiley \& Sons, Hoboken, New Jersey, pp 3-11

Eibl R, Löffelholz C, Eibl D (2011b) Single-use bioreactors-An overview. In: Eibl R, Eibl D (eds) Single-use technology in biopharmaceutical manufacture. Wiley, Hoboken, New Jersey, pp 33-51

Eibl R, Brändli J, Eibl D (2012) Plant cell bioreactors. In: Doelle HW, Rokem S, Berovic M (eds) Biotechnology, Encyclopedia of Life Support Systems (EOLSS). EOLSS Publishers, Oxford, www.eolss.net), [Retrieved] July 24, 2012

Etienne H, Berthouly M (2002) Temporary immersion systems in plant propagation. Plant Cell Tiss Org Cult 69:215-231

Ferri M, Dipalo SCF, Bagni N, Tassoni A (2011) Chitosan elicits mono-glucosylated stilbene production and release in fed-batch bioreactor cultures of grape cells. Food Chem 124:1473-1479
Georgiev MI, Pavlov AI, Bley T (2006) Betalains by transformed Beta vulgaris roots in stirred-tank bioreactor: batch and fed-batch processes. In: Sorvari S, Toldi O (eds) Proceedings of Second International Congress on Bioreactor Technology in Cell, Tissue Cultures and Biomedical Applications. Karhukopio OY, Turku, Finland, pp 22-28

Georgiev MI, Pavlov A, Bley T (2007) Hairy root type plant in vitro systems as sources of bioactive substances. Appl Microbiol Biotechnol 74:1175-1185

Georgiev MI, Georgiev V, Weber J, Bley T, Ilieva M, Pavlov A (2008) Agrobacterium rhizogenes-mediated genetic transformations: A powerful tool for the production of metabolites. In: Wolf T, Koch J (eds) Genetically modified plants. Nova Science Publishers, New York, USA, pp 99-126

Georgiev MI, Abrashev R, Krumova E, Demirevska K, Ilieva M, Angelova M (2009a) Rosmarinic acid and antioxidant enzyme activities in Lavandula vera MM cell suspension culture: A comparative study. Appl Biochem Biotechnol 159:415-425

Georgiev MI, Weber J, Maciuk A (2009b) Bioprocessing plant cell cultures for mass production of targeted compounds. Appl Microbiol Biotechnol 83:809-823

Georgiev MI, Ludwig-Muller J, Bley T (2010) Hairy root culture: copying nature in new bioprocesses. In: Arora R (ed) Medicinal plant biotechnology. CAB International, Oxon, United Kingdom, pp 156-175

Georgiev MI, Ludwig-Muller J, Weber J, Stancheva N, Bley T (2011) Bioactive metabolite production and stress-related hormones in devil's claw cell suspension cultures grown in bioreactors. Appl Microbiol Biotechnol 89:1683-1691

Georgiev MI, Agostini E, Ludwig-Mueller J, Xu J (2012a) Genetically transformed roots: from plant disease to biotechnological resource. Trends Biotechnol 30:528-537

Georgiev V, Ivanov I, Berkov S, Ilieva M, Georgiev MI, Gocheva T, Pavlov A (2012b) Galanthamine production by Leucojum aestivum L. shoot culture in a modified bubble-column bioreactor with internal sections. Eng Life Sci 12:534-543

Girard LS, Fabis MJ, Courtois D, Petiard V, Koprowski H (2006) Expression of a human anti-rabies virus monoclonal antibody in tobacco cell culture. Biochem Biophys Res Commun 345:602-607

Gitzinger M, Parsons J, Reski R, Fussenegger M (2008) Functional cross-kingdom conservation of mammalian and moss (Physcomitrella patens) transcription, translation and secretion machineries. Plant Biotechnol J 7:73-86

Homova V, Weber J, Schulze J, Alipieva K, Bley T, Georgiev MI (2010) Devil's claw hairy root culture in flasks and in a 3-L bioreactor: bioactive metabolite accumulation and flow cytometry. Z Naturforsch $65 \mathrm{c}: 472-478$

Hsiao TY, Bacani FT, Carvalho EB, Curtis WR (1999) Development of a low capital investment reactor system: application for plant cell culture. Biotechnol Prog 15:114-122

Hu FX, Huang JH, Xu YX, Qian XH, Zhong JJ (2006) Responses of defense signals, biosynthetic gene transcription and taxoid biosynthesis to elicitation by a novel synthetic jasmonate in cell cultures of Taxus chinensis. Biotechnol Bioeng 94:1064-1071

Hu WW, Zhong JJ (2001) Effect of bottom clearance on performance of air-lift bioreactor in high-density culture of Panax notoginseng cells. J Biosci Bioeng 92:389-392

Hu WW, Yao H, Zhong JJ (2001) Improvement of Panax notoginseng cell culture for production of ginseng saponin and polysaccharide by high density cultivation in pneumatically agitated bioreactors. Biotechnol Prog 17:838-846

Huang TK, Plesha MA, McDonald KA (2010) Semicontinuous bioreactor production of a recombinant human therapeutic protein using a chemically inducible viral amplicon expression system in transgenic plant cell suspension cultures. Biotechnol Bioeng 106:408-421 
Ivanov I, Georgiev V, Georgiev MI, Ilieva M, Pavlov A (2011) Galanthamine and related alkaloids production by Leucojum aestivum L. shoot culture using a temporary immersion technology. Appl Biochem Biotechnol 163:268-277

Kim YJ, Weathers PJ, Wyslouzil BE (2002) Growth of Artemisia апnиа hairy roots in liquid- and gas-phase reactors. Biotechnol Bioeng 80:454-464

Klöckner W, Raven N, Gacem R, Anderlei T, Büchs J, Schillberg S (2012a) Orbitally-shaken disposable bioreactors - a promising approach for the production of proteins in tobacco cell suspensions. Presentation at GVC/DECHEMA conference, 5/15/2012, Freiburg, Germany

Klöckner W, Tissot S, Wurm F, Büchs J (2012b) Power input correlation to characterize the hydrodynamics of cylindrical orbitally shaken bioreactors. Biochem Eng J 65:63-69

Kretzmer G (2002) Industrial processes with animal cells. Appl Microbiol Biotechnol 59:135-142

Krishnan R, Chen H (2012) A comprehensive strategy to evaluate single-use bioreactors for pilot-scale cell culture production. Am Pharm Rev 15 [Retrieved] November 4, 2012

Kusakari K, Yokoyama M, Inomata S, Gozu Y, Katagiri C, Sugimoto Y (2012) Large-scale production of saikosaponins through root culturing of Bupleurum falcatum L. using modified air-lift reactors. J Biosci Bioeng 113:99-105

Liu YK, Huang LF, Ho SL, Liao CY, Liu HY, Lai YH, Yu SM, Lu CA (2012) Production of mouse granulocyte-macrophage colonystimulating factor by gateway technology and transgenic rice cell culture. Biotechnol Bioeng 109:1239-1247

Liu CZ, Gou C, Wang YC, Ouyang F (2003) Comparison of various bioreactors on growth and artemisinin of Artemisia annua L. shoot cultures. Process Biochem 39:45-49

Liu C, Towler MJ, Medrano G, Cramer CL, Weathers PJ (2009) Production of mouse interleukin-12 is greater in tobacco hairy roots grown in a mist reactor than in an air-lift reactor. Biotechnol Bioeng 102:1074-1086

Ludwig-Mueller J, Georgiev MI, Bley T (2008) Metabolite and hormonal status hairy root cultures of Devil's claw (Harpagophytum procumbens) in flasks and in a bubble-column bioreactor. Process Biochem 43:15-23

Merchuk JC, Gluz M (1999) Bioreactors, air-lift reactors. In: Ficklinger MC, Stephen WD (eds) Encyclopedia of bioprocesss technology: fermentation, biocatalysis, bioseparation, vol 1 . Wiley, New York, pp 320-353

Misawa M (1994) Plant tissue culture: an alternative for production of useful metabolite. FAO Agricultural Services Bulletin, vol. 108

Onrubia M, Cusido RM, Ramirez K, Hernandez-Vazquez L, Moyano E, Bonfill M, Palazon J (2013) Bioprocessing of plant in vitro systems for the mass production of pharmaceutically important metabolites: paclitaxel and its derivates. Curr Med Chem 20 (7):880-891

Paek KY, Murthy HN, Hahn EJ, Zhong JJ (2009) Large scale culture of ginseng adventitious roots for production of ginsenosides. Adv Biochem Eng Biotechnol 113:151-176

Palazon J, Mallol A, Eibl R, Lettenbauer C, Cusido RM, Pinol MT (2003) Growth and ginsenoside production in hairy root cultures of Panax ginseng using a novel bioreactor. Planta Med 69:344-349

Park CI, Lee SJ, Kang SH, Jung HS, Kim DI, Lim SM (2010) Fed-batch cultivation of transgenic rice cells for the production of hCTLA4Ig using concentrated amino acids. Process Biochem 45:67-74

Pavlov A, Georgiev MI, Ilieva M (2005) Production of rosmarinic acid by Lavandula vera MM cell suspension in bioreactor: effect of dissolved oxygen concentration and agitation. World J Microbiol Biotechnol 21:389-392

Pavlov A, Georgiev MI, Bley T (2007) Batch and fed-batch production of betalains by Red beet (Beta vulgaris) hairy roots in a bubblecolumn reactor. Z Naturforsch 62c:439-446
Perez JAS, Porcel EMR, Lopez JLC, Sevilla JMF, Chisti Y (2006) Shear rate in stirred-tank and bubble-column bioreactors. Chem Eng J 124:1-5

Prakash G, Srivastava AK (2007) Azadirachtin production in stirredtank reactors by Azadirachta indica suspension culture. Process Biochem 42:93-97

Prakash G, Srivastava AK (2011) Integrated yield and productivity enhancement strategy for biotechnological production of Azadirachtin by suspension culture of Azadirachta indica. AsiaPacific J Chem Eng 6:129-137

Pietrzykowski M, Flanagan W, Pizzi V, Brown A, Sinclair A, Monge M (2011) An environmental life-cycle assessment comparing single-use and conventional process technology. BioPharm Int 24:30-38

Qian ZG, Zhao ZJ, Xu YF, Qian XH, Zhong JJ (2004) Novel chemically synthesized hydroxyl-containing jasmonates as powerful inducing signals for plant secondary metabolism. Biotechnol Bioeng 86:809-816

Qian ZG, Zhao ZJ, Xu YF, Qian XH, Zhong JJ (2005) A novel synthetic fluoro-containing jasmonate derivative acts as a chemical inducing signal for plant secondary metabolism. Appl Microbiol Biotechnol 68:98-103

Rader R, Langer E (2012) Upstream single-use bioprocessing systems - future market trends and growth assessment. BioProcess Int 10:5

Ramakrishnan D, Curtis WR (2004) Trickle-bed root culture bioreactor design and scale-up: growth, fluid-dynamics and oxygen mass transfer. Biotechnol Bioeng 88:248-260

Rao G, Moreira A, Brorson K (2009) Disposable bioprocessing: the future has arrived. Biotechnol Bioeng 102:348-356

Raposo S, Lima-Costa ME (2012) Effects of the hydrodynamic environment and oxygen mass transfer on plant cell growth and milkclotting protease production in a stirred-tank reactor. Eng Life Sci 12:441-449

Raven N, Schillberg S, Kirchhoff J, Brändli J, Imseng N, Eibl R (2011) Growth of BY-2 suspension cells and plantibody production in single-use bioreactors. In: Eibl R, Eibl D (eds) Single-use technology in biopharmaceutical manufacture. Wiley, Hoboken, New Jersey, pp 251-261

Ritala A, Wahlström EH, Holkeri H, Hafren A, Mäkeläinen K, Baez J, Mäkinen K, Nuutila AM (2008) Production of a recombinant industrial protein using barley cell cultures. Protein Expres Purif 59:274-281

Roberts ML, Herrera-Herrera JL, Herrera-Herrera G, Herrera-Alamillo MA, Fuentes-Carillo PF (2006) A new temporary immersion bioreactor system for micropropagation. In: Loyola-Vargas VM, Vazquez-Flota F (eds) Plant cell culture protocols. Humana Press, Totowa, New Jersey, pp 121-129

Routien JB, Nickell LG (1956) Cultivation of plant tissue. US Patent 2:747,334

Sajc L, Grubisic D, Vunjak-Novakovic G (2000) Bioreactors for plant engineering: an outlook for further research. Biochem Eng J 4:89 99

Schürch C, Blum P, Zülli F (2008) Potential of plant cell culture for cosmetic application. Phytochem Rev 7:599-605

Shaaltiel Y, Kirshner Y, Shtainiz A, Naos Y, Shneor Y (2010) Large scale disposable bioreactor. EP 2150608 A2

Sivakumar G, Liu C, Towler MJ, Weathers PJ (2010) Biomass production of hairy roots of Artemisia annua and Arachis hypogaeae in a scaled-up mist bioreactor. Biotechnol Bioeng 107:802-813

Vanhamel S, Masy C (2011) Production of disposable bags: A manufacturer's report. In: Eibl R, Eibl D (eds) Single-use technology in biopharmaceutical manufacture. Wiley, Hoboken, New Jersey, pp 113-134

Veliky IA, Martin SM (1970) A fermenter for plant cell suspension cultures. Can J Microbiol 16:223-226 
Verpoorte R, Contin A, Memelink J (2002) Biotechnology for the production of plant secondary metabolites. Phytochem Rev $1: 13-25$

Wang SJ, Zhong JJ (1996a) A novel centrifugal impeller bioreactor. I. Fluid circulation, mixing, and liquid velocity profiles. Biotechnol Bioeng 51:511-519

Wang SJ, Zhong JJ (1996b) A novel centrifugal impeller bioreactor. II. Oxygen transfer and power consumption. Biotechnol Bioeng 51:520-527

Watt MP (2012) The status of temporary immersion system (TIS) technology for plant micropropagation. Afr J Biotechnol 11:14025-14035

Weathers PJ, Towler MJ, Xu JF (2010) Bench to batch: advances in plant cell culture for producing useful products. Appl Microbiol Biotechnol 85:1339-1351

Werner S, Eibl R, Lettenbauer C, Röll M, Eibl D, DeJesus M, Zhang X, Stettler M, Tissot S, Bürki C, Broccard G, Kühner M, Tanner R, Baldi L, Hacker D, Wurm FM (2010) Innovative, non-stirred bioreactors in scales from milliliters up to 1000 Liters for suspension cultures of cells using disposable bags and containers-a Swiss contribution. Chimia 64:819-823

Werner S, Eibl D, Olownia J, Egger D (2012) 2-D orbitally shaken single-use bags: Engineering characterization and cell culture application examples. Presentation at GVC/DECHEMA conference, 5/15/2012, Freiburg, Germany

Werner S, Olownia J, Egger D, Eibl D (2013) Scale-up rules for orbitally shaken single-use cultivation systems being geometrically similar and non-similar. Chem Ingen Tech doi:10.1002/ cite201200153

Wilson SA, Roberts SC (2012) Recent advances towards development and commercialization of plant cell culture processes for the synthesis of biomolecules. Plant Biotechnol J 10:249-268

Wink M, Alfermann AW, Franke R, Wetterauer B, Distl M, Windhoevel J, Krohn O, Fuss E, Garden H, Mohagheghzadeh A, Wildi E, Ripplinger P (2005) Sustainable production of phytochemicals by plant in vitro cultures: anticancer agents. Plant Gene Res 3:90-100
Yue XG, Zhang W, Deng MC (2011) Hyper-production of C-13labeled trans-resveratrol in Vitis vinifera suspension cell culture by elicitation and in situ adsorption. Biochem Eng J 53:292-296

Zhang ZY, Zhong JJ (2004) Scale-up of centrifugal impeller bioreactor for hyperproduction of ginseng saponin and polysaccharide by high-density cultivation of Panax notoginseng cells. Biotechnol Prog 20:1076-1081

Zhong JJ, Fujiyama K, Seki T, Yoshida T (1994) A quantitative analysis of shear effects on cell suspension and cell culture of Perilla frutescens in bioreactors. Biotechnol Bioeng 44:649-654

Zhong JJ (2001) (ed.) Plant cells. Adv Biochem Eng/Biotechnol, vol. 72. Springer-Verlag, Heidelberg

Zhong JJ (2002) Plant cell culture for production of paclitaxel and other taxanes. J Biosci Bioeng 94:591-599

Zhong JJ, Pan ZW, Wang ZY, Wu JY, Chen F, Takagi M, Yoshida T (2002) Effect of mixing time on taxoid production in suspension cultures of Taxus chinensis in a centrifugal impeller bioreactor. J Biosci Bioeng 94:244-250

Zhong JJ (2011) Bioreactor Engineering. In: Murray M-Y (ed.), Comprehensive Biotechnology, Second Edition, vol. 2. Elsevier, pp. $165-177$

Zhou TC, Zhou WW, Hu W, Zhong JJ (2010) Cell culture, bioreactors, commercial production. Encyclopedia of Industrial Biotechnology, vol. 2. Wiley, pp. 913-939

Zhou X, Zhong JJ (2011a) Quantitative influence of endogenous salicylic acid level on taxuyunnanine $C$ biosynthesis in suspension cultures of Taxus chinensis. Biotechnol Bioeng 108:216-221

Zhou X, Zhong JJ (2011b) Intracellular salicylic acid is involved in signal cascade regulating low ammonium induced taxoid biosynthesis in suspension cultures of Taxus chinensis. Appl Microbiol Biotechnol 90:1027-1036

Ziv M, Ronen G, Raviv M (1998) Proliferation of meristematic clusters in disposable pre-sterilized plastic biocontainers for the largescale micropropagation of plants. In Vitro Cell Dev Biol Plant $34: 152-158$

Ziv M (2005) Bioreactor technology for plant micropropagation. Hort Rev 24:1-30 